\title{
Short-term results of hip revisions with a curved cementless modular stem in association with the surgical approach
}

\author{
Bernd Fink • Alexandra Grossmann • \\ Svenja Schubring $\cdot$ Martin S. Schulz $\cdot$ Martin Fuerst
}

Published online: 26 November 2008

(C) Springer-Verlag 2008

\section{Erratum to: Arch Orthop Trauma Surg}

\section{DOI 10.1007/s00402-008-0617-7}

There was an error in the column heads of Table 2, where

"Trendelenburg limping" appeared instead of the correct

"Trendelenburg sign".

The corrected table is given in full here.

Table 2 Harris Hip Score und frequency of Trendelenburg signs in Revitan Curved stems implanted endofemoral and transfemoral

\begin{tabular}{|c|c|c|c|c|c|c|}
\hline \multirow[t]{2}{*}{ Time } & \multicolumn{3}{|c|}{ Harris Hip Score } & \multicolumn{3}{|c|}{ Trendelenburg sign } \\
\hline & $\begin{array}{l}\text { Endofem. } \\
N=42\end{array}$ & $\begin{array}{l}\text { Transfem. } \\
N=78\end{array}$ & $\begin{array}{l}P \text { value } \\
\text { Mann-Whitney }\end{array}$ & $\begin{array}{l}\text { Endofem. } \\
N=42\end{array}$ & $\begin{array}{l}\text { Transfem. } \\
N=78\end{array}$ & $\begin{array}{l}P \text { value } \\
\text { Chi }^{2} \text { test }\end{array}$ \\
\hline Preoperative & $48.3 \pm 15.5$ & $44.1 \pm 16.3$ & $P=0.226$ & $23(54.8 \%)$ & $43(55.1 \%)$ & $P=0.969$ \\
\hline 3 months & $72.2 \pm 14.9$ & $61.0 \pm 14.3$ & $P<0.001$ & $28(66.6 \%)$ & $68(87.2 \%)$ & $P=0.007$ \\
\hline 6 months & $83.6 \pm 19.9$ & $72.1 \pm 15.1$ & $P=0.001$ & $21(50 \%)$ & $58(74.3 \%)$ & $P=0.007$ \\
\hline 9 months & $91.4 \pm 11.3$ & $78.8 \pm 18.6$ & $P=0.001$ & $18(42.8 \%)$ & $51(65.4 \%)$ & $P=0.017$ \\
\hline 12 months & $92.3 \pm 11.6$ & $81.4 \pm 16.9$ & $P=0.002$ & $18(42.8 \%)$ & $49(62.8 \%)$ & $P=0.035$ \\
\hline 18 months & $92.2 \pm 12.8$ & $84.7 \pm 15.3$ & $P=0.046$ & $15(35.7 \%)$ & $45(57.7 \%)$ & $P=0.021$ \\
\hline 24 months & $92.9 \pm 12.1$ & $86.3 \pm 15.6$ & $P=0.067$ & $11(26.2 \%)$ & $41(52.6 \%)$ & $P=0.005$ \\
\hline
\end{tabular}

The online version of the original article can be found under doi:10.1007/s00402-008-0617-7.

B. Fink $(\bowtie) \cdot$ A. Grossmann · M. S. Schulz

Clinic of Joint Replacement,

General and Rheumatic Orthopaedics,

Orthopaedic Clinic Markgröningen gGmbH,

Kurt-Lindemann-Weg 10, 71706 Markgröningen, Germany

e-mail: b.fink@okm.de

\section{S. Schubring $\cdot$ M. Fuerst}

Orthopaedic Department of Rheumaklinik Bad Bramstedt,

Oskar-Alexander-Str. 26, 24576 Bad Bramstedt, Germany 